\title{
Commentary: A bandage for dilated left ventricular cardiomyopathy
}

\author{
Aaron Eckhauser, MD, MS
}

\author{
From the Division of Cardiothoracic Surgery, Department of Surgery, University of Utah, Salt Lake City, Utah. \\ Disclosures: Author has nothing to disclose with regard to commercial support. \\ Received for publication Feb 4, 2019; accepted for publication Feb 4, 2019; available ahead of print March 20, \\ 2019. \\ Address for reprints: Aaron Eckhauser, MD, MS, 100 N Mario Capecchi Dr, Suite 2200, Salt Lake City, UT 84113 \\ (E-mail: Aaron.eckhauser@hsc.utah.edu). \\ J Thorac Cardiovasc Surg 2019;157:2431-2 \\ $0022-5223 / \$ 36.00$ \\ Copyright (C) 2019 by The American Association for Thoracic Surgery \\ https://doi.org/10.1016/j.jtcvs.2019.02.015
}

In this month's edition of The Journal of Thoracic and Cardiovascular Surgery, Yerebakan and colleagues ${ }^{1}$ present their work studying the effects of central pulmonary artery banding on doxorubicin-induced left ventricular (LV) cardiomyopathy in juvenile animals. Their group used selective left main intracoronary injections of doxorubicin in 4-month-old sheep to induce LV dilation and impairment and then subjected the animals to either central pulmonary artery banding (CPAB) or a sham operation. Echocardiography conducted before the animals were killed and the hearts explanted demonstrated that CPAB improved the LV function and reduced the LV dimensions without an effect on the pressure-volume loop assessments. Although this preliminary work contains several limitations, the therapeutic implications remain significant; namely, the work suggests the novel use of CPAB to treat the different etiologies leading to LV functional compromise with dilation in children (ie, idiopathic, toxic, or ischemic cardiomyopathy).

The initial hypotheses of Yerebakan and colleagues ${ }^{1}$ were partially informed by work performed by Schranz and associates, ${ }^{2}$ who studied the use of CPAB to treat end-stage LV dilated cardiomyopathy with preserved right ventricular (RV) function. Schranz and associates ${ }^{2}$ reported a worldwide experience that spanned the course of 10 years in which this therapy was used in 70 patients with a median follow-up of just more than 3 years. Forty-two patients had a positive response to the CPAB therapy, with 34 patients achieving functional recovery (27 of whom ultimately underwent debanding) and 8 patients demonstrating progressive but clinically incomplete recovery. Schranz and associates ${ }^{2}$ hypothesized that an acute increase in RV afterload would help to reestablish interventricular septal position and geometry, thus improving LV function, and also that acute RV in this study.

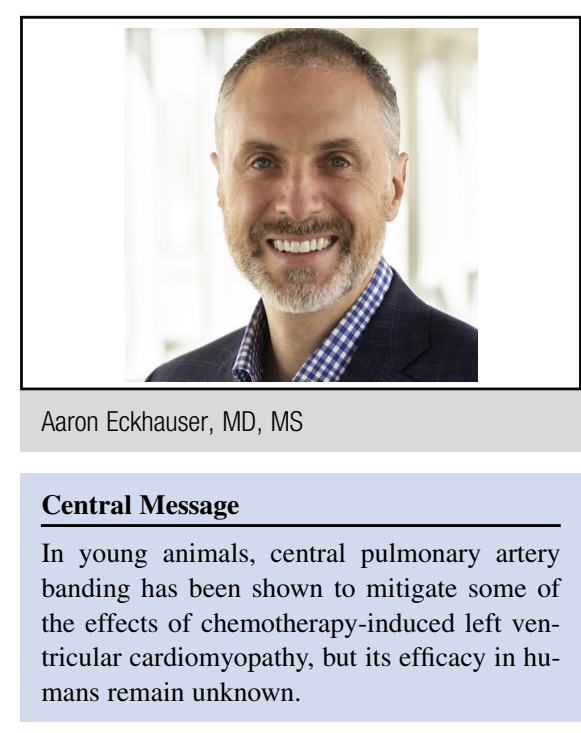

See Article page 2416.

hypertrophy could potentially promote cardiac regeneration, leading to cardiac improvement.

Although the design and execution of this study are scientifically sound, there are several important limitations that caution against a broader application of these findings without further study. First, the echocardiographic improvements in LV function and size were not mirrored by similar improvements in symptomatic and hemodynamic outcomes, calling into question the true clinical implications of CPAB in this cohort. In addition, these animals were killed after a median of 3 months, a relatively short period of follow-up, and insight into the medium and long-term effects of this therapy is therefore lacking. Second, the CPAB cohort was compared with a cohort of subjects undergoing a sham operation. In reality, we choose between optimal medical heart failure therapies in addition to or in place of surgical therapy (heart transplant or mechanical circulatory support). It would be extremely important to understand how the CPAB cohort would compare with a similar cohort of animals that received full medical therapy. Third, the potential regenerative effects of RV hypertrophy were not specifically investigated

Despite these noted limitations, and the rigorous work required for further study, I maintain a tempered 
enthusiasm toward the potential of this therapy to enhance our ability to treat pediatric patients with dilated LV cardiomyopathies. I congratulate Yerebakan and colleagues ${ }^{1}$ for enhancing our study and understanding of the many nuances of advanced heart failure in children.

\section{References}

1. Yerebakan C, Boltze J, Elmontaser H, Yoruker U, Latus H, Khalil M, et al. Effects of pulmonary artery banding in doxorubicin-induced left ventricular cardiomyopathy. J Thorac Cardiovasc Surg. 2019;157:2416-28.e4.

2. Schranz D, Akintuerk H, Bailey L. Pulmonary artery banding for functional regeneration of end-stage dilated cardiomyopathy in young children. Circulation. 2018; 137:1410-2. 